\title{
Haplosyllis xeniaecola, ein neuer Polychaet (Syllidae) von den Molukken (Indonesien)
}

\author{
Gesa Hartmann-Schröder* \\ Biologische Anstalt Helgoland (Zentrale) Notkestr. 31, D-22607 Hamburg, \\ Bundesrepublik Deutschland \\ Zoologisches Institut und Zoologisches Museum der Universität Hamburg, Martin- \\ Luther-King-Platz 3, D-20146 Hamburg, Bundesrepublik Deutschland * *
}

\begin{abstract}
Haplosyllis xeniaecola a new polychaete species (Syllidae) from the Molukken Islands (Indonesia). Haplosyllis xeniaecola sp. $\mathrm{n}$. is described. It was collected by W. Kükenthal from Xenia viridis (Octocorallia) at Ternate, Molukken Islands.
\end{abstract}

\section{EINLEITUNG}

Im Jahre 1894 sammelte W. Kükenthal auf einer zoologischen Forschungsreise zu den Molukken und nach Borneo unter anderem eine Oktokoralle, die zwei Jahre später von Schenk als neue Art Xenia viridis Schenk, 1896 (Xeniidae, Alcyonaria) beschrieben wurde. Bei seinen Untersuchungen an Xeniiden des Roten Meeres ließ sich Götz Reinicke, Institut für Hydrobiologie der Universität Essen, den Holotypus von Xenia viridis aus dem Forschungsinstitut Senckenberg in Frankfurt zuschicken. Bei dieser Gelegenheit entdeckte Reinicke einen Polychaeten an der Koralle und bat mich darauf hin, diesen Polychaeten zu bestimmen. Dabei stellte es sich heraus, daß es sich um eine neue Art der Gattung Haplosyllis Langerhans, 1879 handelt.

\section{BESCHREIBUNG DER ART}

Familie Syllidae

Gattung Haplosyllis Langerhans, 1879

Haplosyllis xeniaecola sp. $\mathrm{n}$.

(Abb. 1-8)

Holotypus mit 111 Borstensegmenten $15 \mathrm{~mm}$ lang; größte Breite ohne Parapodien $0,75 \mathrm{~mm}$. Keine besondere Färbung (nach fast 100 Jahren in Alkohol). Körperoberfläche glatt, mit winzigen "Pünktchen" (Drüsen?) in der Epidermis; dazu Segmente hinter dem Magen mit fünf bis sechs Querlinien, Segmente dadurch wie sekundär geringelt erschei-

- Mitglied der Taxonomischen Arbeitsgruppe an der Biologischen Anstalt Helgoland

* Korrespondenzanschrift 
nend. Hintere 22 Segmente etwas abgesetzt und innen dunkler (beginnende StolonBildung?).

Prostomium breiter als lang, mit vier jetzt farblosen Augen im Trapez. Mediane Antenne etwa sechsmal so lang wie das Prostomium, auf der Mitte des Prostomiums inserierend; beiderseits davon eine V-förmige Falte auf dem Prostomium (Abb.1). Laterale Antennen am Vorderrand des Prostomiums, etwa halb so lang wie die mediane. Antennen glatt, nur distal undeutlich geringelt. Palpen als breite Lappen, die völlig voneinander getrennt und leicht ventrad umgebogen.

1. Segment deutlich vom Prostomium abgesetzt; Vorderkörper sonst ohne deutliche Segmentgrenzen. Dorsale Tentakelcirren etwas kürzer als die mediane Antenne, die ventralen etwas mehr als halb so lang wie die dorsalen; beide in der Form wie die Antennen. Parapodien kurz, abgestutzt, vorn mit fünf, danach mit drei bis vier und hinten mit zwei nadelförmigen hellbraunen Aciculae. Dorsalcirren in der Länge alternierend: die langen entsprechen etwas mehr als der doppelten Segmentbreite, die kürzeren sind

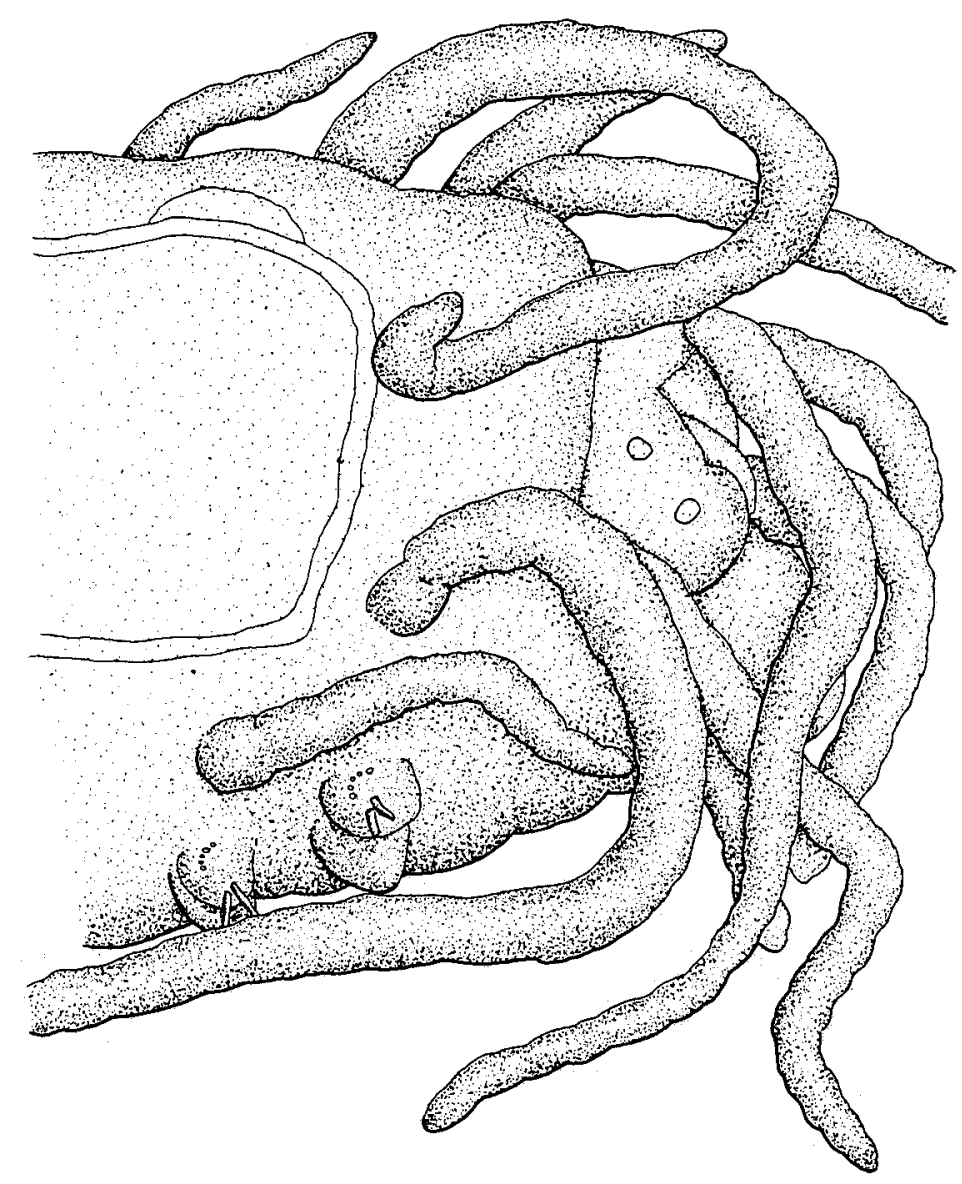

Abb. 1. Haplosyllis xeniaecola sp. n. Vorderende von dorsolateral 
etwas mehr als halb so lang wie die langen. Ventralcirren zungen- bis fingerförmig, meist etwas kürzer, in der Körpermitte etwa gleich lang wie der Parapodiallappen.

Borsten alle einfach, kurz und kräftig, distal mehr oder weniger hakig gebogen, einspitzig und subdistal unterhalb des konkaven Abschnitts mit mehr oder weniger ausgeprägtem Höcker; einige Borsten auch unterhalb des konvexen Abschnitts mit Andeutung eines Höckers oder eines Knicks (Abb. 2-8). Vordere zwei Parapodienpaare mit zwei oder drei Borsten, danach unregelmäßig drei oder vier Borsten. Borsten im Parapod jeweils etwas unterschiedlich in der Größe und in der Form (Abb. 2-8).

Pygid mit zwei langen Analcirren mit etwa 20 undeutlichen Gliedern oder Ringelungen. Pharynx über vier Segmente reichend, relativ breit; Zahn nicht genau zu erkennen. Magen über sechs Segmente, mit 30 Muskelzellreihen.

L o c u s ty p ic u s: Ternate (Molukken), von Xenia viridis Schenk, 1896 (Xeniidae, Alcyonaria, Octocorallia); keine weitere Information vorhanden: Holotypus im Forschungsinstitut Senckenberg in Frankfurt (SMF:4433).

Derivatio nominis: xeniaecola = Bewohner von Xenia.

Taxonomische Bemerkung: In der Familie Syllidae besitzen drei Gattungen nur einfache Borsten; zusammengesetzte Borsten fehlen: Haplosyllis, Geminosyllis Imajima, 1966 und die Untergattung Trypanobia Imajima \& Hartmann, 1964 der Gattung

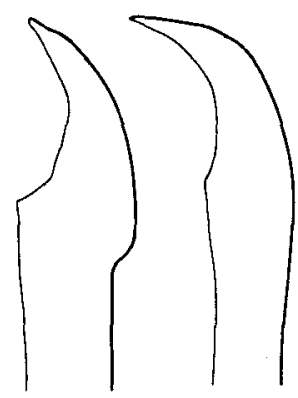

Abb. 2. Haplosyllis xeniaecola sp. n. Borsten aus 2. Parapod

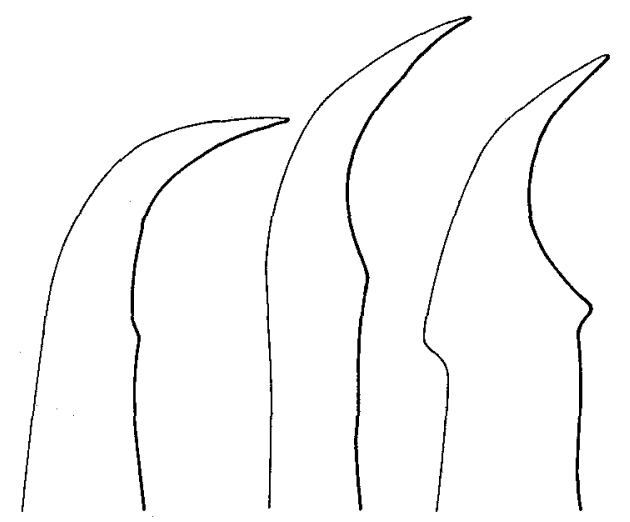

Abb. 3. Haplosyllis xeniaecola sp. n. Borsten aus 9. Parapod 

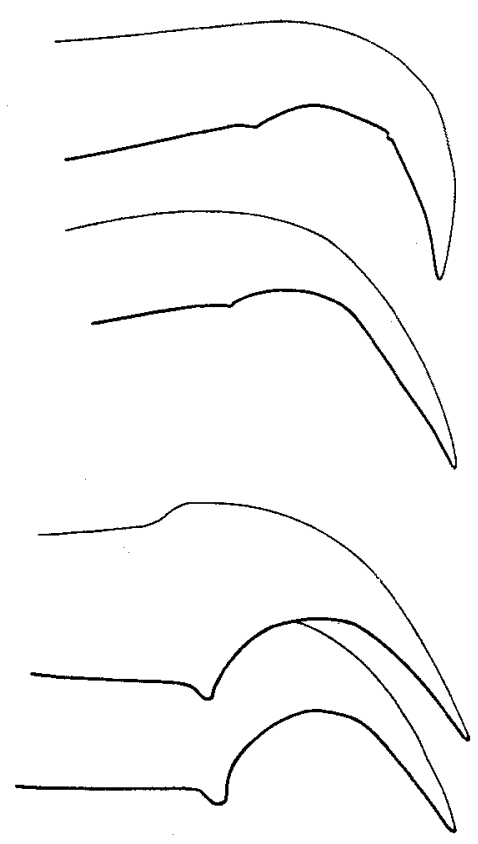

Abb. 4. Haplosyllis xeniaecola sp. n. Borsten aus 15. Parapod

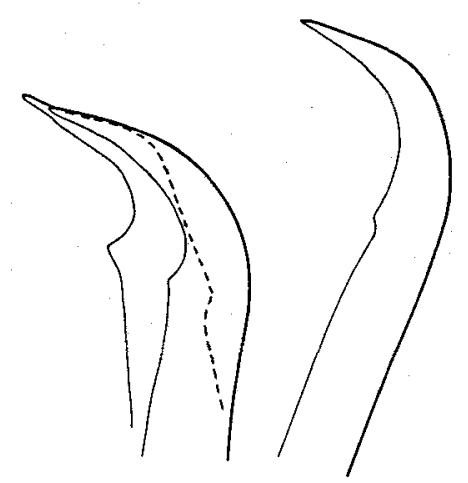

Abb. 5. Haplosyllis xeniaecola sp. n. Borsten aus 35. Parapod

Trypanosyllis. Abgesehen vom Trepan am Eingang des Rüssels haben die Arten der Gattungen Geminosyllis und Trypanosyllis (Trypanobia) unten im Borstenbündel eine feine einfache Borste, die der unteren einfachen Borste der Typosyllis-Arten entspricht. Diese einfache Borste fehlt bei Haplosyllis und der hier neu beschriebenen Art. Aus diesem Grunde wird sie der Gattung Haplosyllis zugeordnet. Darüber hinaus sind alle bisher bekannten Haplosyllis-Arten Kommensalen mit anderen Wirbellosen, Schwämmen, Korallen und Echinodermen. Von Octocorallia sind $H$. bisetosa Hartmann-Schrö- 


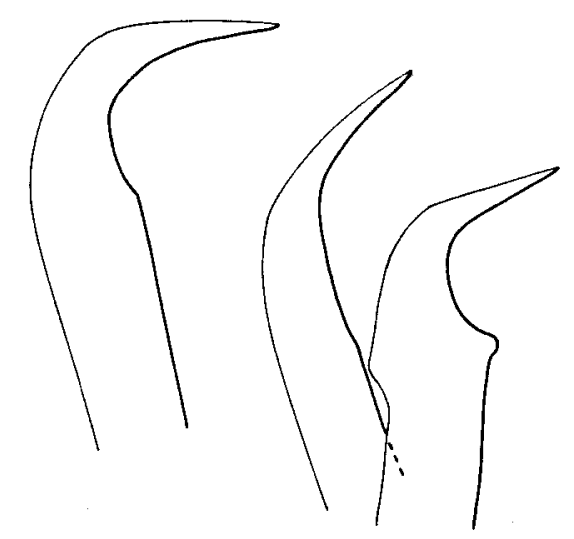

Abb. 6. Haplosyllis xeniaecola sp. n. Borsten aus 70. Parapod

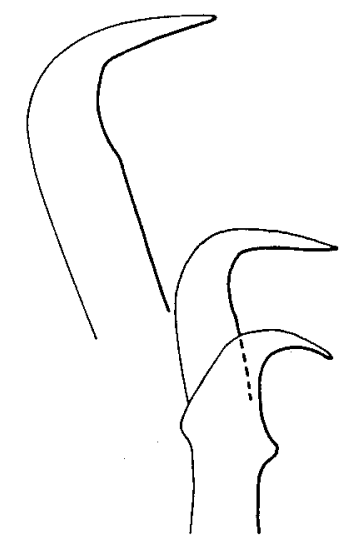

Abb. 7. Haplosyllis xeniaecola sp. n. Borsten aus 90. Parapod

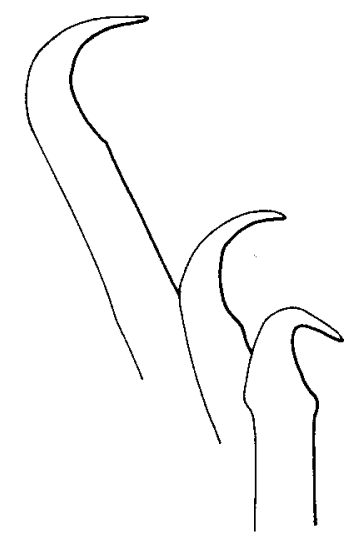

Abb. 8. Haplosyllis xeniaecola sp. n. Borsten aus 100. Parapod 
der, 1960 und $H$. chamaeleon Laubier, 1960 gefunden worden, letztere vermutlich als Ektoparasit. Von diesen Arten unterscheidet sich die neue Art sowohl durch die anders geformten Borsten als auch durch die Cirren, die bei $H$. bisetosa an der Basis, bei $H$. chamaeleon vollständig und deutlich gegliedert sind. Auch die übrigen Arten der Gattung weisen deutliche Unterschiede besonders in der Form der Borsten auf (vergl. Tabelle 1 in Hartmann-Schröder, 1978). Auch eine neue Art aus China, die von Sun beschrieben wird (pers. Komm.), differiert deutlich in der Form der Borsten und in der Form der deutlich gegliederten Cirren und Antennen.

\section{LITERATUR}

Hartmann-Schröder, G., 1960. Polychaeten aus dem Roten Meer. - Kieler Meeresforsch. 16, 69-125. Hartmann-Schröder, G., 1978. Einige Sylliden-Arten (Polychaeta) von Hawaii und aus dem Karibischen Meer. - Mitt. hamb. zool. Mus. Inst. 75, 49-61.

Imajima, M., 1966. The Syllidae (Polychaetous annelids) from Japan. (IV) Syllinae (1). - Publ. Seto mar. biol. Lab. 14, 219-252.

Imajima, M. \& Hartmann, O., 1964. The polychaetous annelids of Japan (Part I). - Occ. Pap. Allan Hancock Fdn. Publs. 26, 1-237.

Laubier, L, 1960. Une nouvelle sous-espèce de Syllidiens: Haplosyllis depressa Augener chamaeleon n. subsp., ectoparasite sur l'Octocoralliaire Muricea chamaeleon von Koch. - Vie Milieu 11, 75-87.

Schenk, A., 1896. Clavulariiden, Xeniiden und Alcynoiiden von Ternate, - Abh. senckenb. naturf. Ges. 23(1), 40-80. 